\title{
Continuous muscle fibre activity: a case treated with acetazolamide
}

\author{
Neşe Çelebisoy, Zafer Çolakoğlu, Yaprak Akbaba, Nur Yüceyar
}

\begin{abstract}
A case is reported of the continuous muscle fibre activity syndrome, which includes a group of disorders characterised by sustained motor unit activity due to hyperactivity of peripheral nerve motor axons. In this patient the muscle stiffness and myokymic movements were successfully treated with acetazolamide, which acts as a membrane stabiliser either by blockade of chloride and bicarbonate membrane transport or by producing kaliuresis and raising the transmembrane potential by decreasing extracellular potassium.
\end{abstract}

(F Neurol Neurosurg Psychiatry 1998;64:256-258)

Keywords: continuous muscle fibre activity syndrome; acetazolamide

Continuous muscle fibre activity and the eponym Isaacs' syndrome describes a heterogeneous group of conditions that exhibit sustained, diffuse motor unit activity due to hyperactivity of peripheral nerve motor axons. ${ }^{1-3}$ These conditions can either be associated with clinical or EMG evidence of peripheral neuropathy or not and generally respond to either phenytoin or carbamazepine, which are voltage dependent sodium channel blockers. ${ }^{4}$

\section{Case report}

A 38 year old woman, who was diagnosed as having Isaacs' syndrome 12 years ago and treated with $200 \mathrm{mg}$ carbamazepine three times

Ege University, Faculty of Medicine,

Department of

Neurology, Bornova,

Izmir, 35100, Turkey

$\mathrm{N}$ Çelebisoy

Z Çolakoğlu

Y Akbaba

N Yüceyar

Correspondence to: Dr Nese Celebisoy, Ege University Faculty of Medicine, Department of Neurology, Bornova, Izmir 35100, Turkey. Telephone 00232 3880980; fax 00232 3422141 .

Received 14 May 1997 and in revised form 21 July 1997 Accepted 29 July 1997

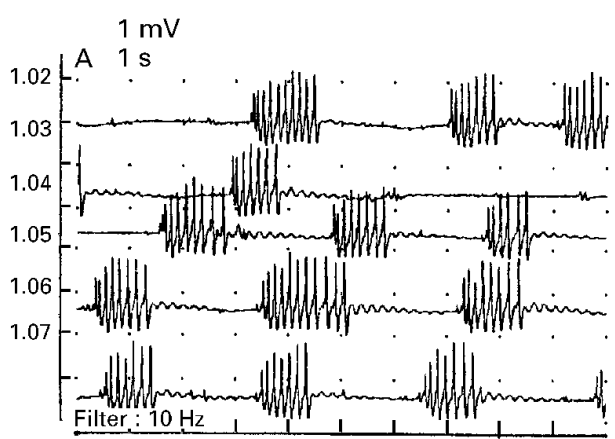

daily, was admitted to hospital because of an increase in cramps and muscle stiffness. On examination, the muscles were stiff with generalised myokymia, more prominent in distal lower limbs. Calf muscles were hypertrophic with bilateral pes cavus and hammer toe deformities. The reflexes were lost bilaterally in the lower limbs. The remainder of the examination was normal. Her medical history was insignificant. Needle EMG showed continuous motor unit activity with myokymic discharges. After repetitive stimulation of the motor nerves, after-discharges were recorded. Nerve conduction studies showed segmental demyelination, more prominent in motor nerves. The function of the motor end plates was normal. Complete blood count, sedimentation rate, routine blood biochemical analysis, protein and immune electrophoresis, antinuclear antibody, anti-DNA, and rheumatoid factor were in the normal range. No abnormality could be detected in abdominal and pelvic ultrasonography and in thorax CT. On admission, carbamazepine was stopped and $25 \mathrm{mg}$ lamotrigine; also a sodium channel blocker, was given daily. After a week, the patient refused to use the drug as cramps, generalised twitching, and muscle stiffness became very disturbing. The next drug tried was mexiletine. She was given $200 \mathrm{mg}$ twice daily for one week. The patient reported it to be better than lamotrigine but not as good as carbamazepine. Acetazolamide was the third choice and a dramatic relief was reported by the patient after doses of $250 \mathrm{mg}$ twice daily. The muscle stiffness

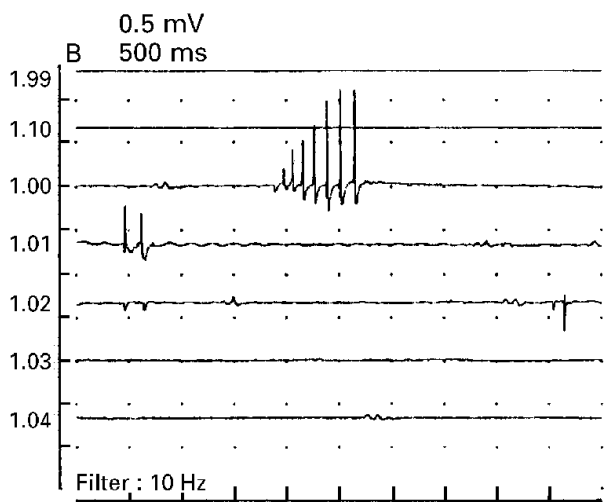

Figure 1 (A) Bursts of repetitively firing motor unit potentials recorded from the soleus muscle during treatment with carbamazepine. (B) Supression of the activity during treatment with acetazolamide. 

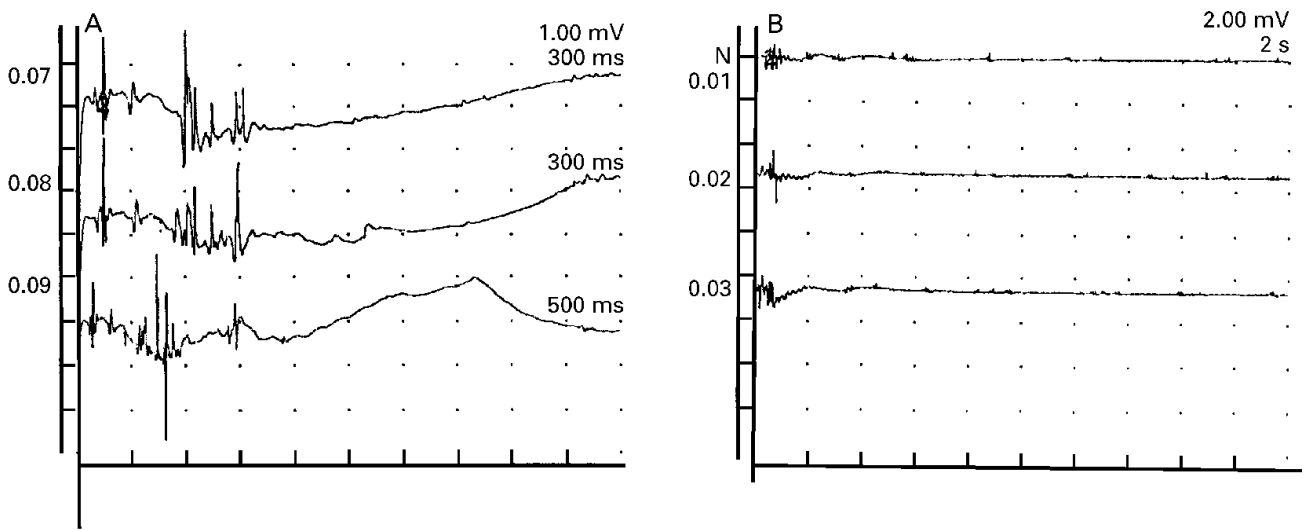

Figure 2 (A) After-discharges recorded from the soleus muscle by stimulation of the tibial nerve at the knee during treatment with carbamazepine. (B) Disappearance of the after-discharges during treatment with acetazolamide.

regressed and myokymic movements became nearly invisible. In the needle EMG, the continuous motor unit activity was supressed (fig 1) and the after-discharges recorded after motor nerve stimulation had disappeared (fig 2). The patient used the drug for three weeks with appreciable recovery, but found acral paraesthesia too disturbing to continue and asked to change back to carbamazepine. She is now back on carbamazepine therapy.

Acetazolamide is an unsubstituted sulphonamide. Its main biochemical action is the inhibition of carbonic anhydrase, an enzyme distributed widely in the brain and other tissues. Besides being a diuretic agent, it is used in diverse neurological disorders. Carbonic anhydrase is found in glial cells and myelin. Its inhibition in the brain causes a rise in total brain carbon dioxide concentrations and also a blockade of chloride and bicarbonate membrane transport and increases the transmembrane chloride gradient. This is its mechanism of action in epilepsy. ${ }^{5}$ It is used in the prophylactic treatment of hypokalamic periodic paralysis. The mechanism of action is uncertain. Because acidosis retards the influx of potassium into cells, the metabolic acidosis produced by acetazolamide has been postulated to be the mechanism of action in hypokalaemic periodic paralysis..$^{6-8}$ On the other hand, acetazolamide may produce a non-acidosis dependent alteration of muscle membrane function that results in a decreased net flux of potassium into muscle. This alteration of muscle membrane function may relate in part to acetazolamide's beneficial effect in hypokalaemic periodic paralysis. ${ }^{9}$ Acetozolamide responsive myotonia congenita, first described by Trudell et $a l^{10}$ resembles Thomsen's disease in appearance except for painful muscle stiffness. ${ }^{11}$ Myotonia is provoked by fasting and oral potassium and relieved by carbohydrate, similar to hyperkalaemic paralysis, although paralysis is not a feature. Acetazolamide tends to provide dramatic relief. An increase in the ratio of extracellular to intracellular potassium would be expected to decrease the muscle fibre transmembrane potential and render it more liable to depolarisation. Acetazolamide administration produces a kaliuresis and decrease in extracellular potassium and may raise the mus- cle transmembrane potential. ${ }^{10}$ Familial ataxia and myokymia is another hereditary disorder in which acetazolamide is used. Brunt et $a l^{12}$ found acetazolamide to be effective in suppressing the ataxic episodes, although there was an increase in continuous motor unit activity. In a patient described by Vaamande et $a l^{13}$ acetazolamide was of no therapeutic value in suppressing the ataxic episodes. Molecular genetic advantages have led to identification of two distinct autosomal dominant disorders characterised by episodic ataxia; episodic ataxia type $I$ is caused by mutations in the potassium channel gene (KCNA 1) on chromosome $12 .{ }^{14}$ The cause of episodic ataxia type II is not yet identified. The effect of acetazolamide in the prevention of ataxic episodes is related to its negative effect on neuronal excitability which is non-specific. ${ }^{12}$ The mechanism leading to hyperactivity of the peripheral nerves in continuous muscle fibre activity is not clear. Sinha et $a l^{15}$ and Newsom-Davis and Mills ${ }^{16}$ postulated that the increased nerve terminal excitability could be due to interference with function of slowly activating potassium channels that normally stabilise the membrane potential. Antibodies to voltage gated potassium channels have been detected in some patients. ${ }^{17}$ Phenytoin and carbamazepine is effective in most, but not all, patients. This effect is due to reduced flux of sodium ions during action potentials. ${ }^{4}$ Mexiletine; a sodium channel blocker, also proved to be effective. ${ }^{18}$ Acetazolamide was first used by Mertens and Zschoke $^{19}$ in cases of neuromyotonia and a reduction in resting muscle activity was detected. On the other hand, Brunt et all found that there was an increase in continuous muscle fibre activity in patients with familial ataxia and myokymia when acetazolamide was given. In our patient a dramatic relief was found with acetazolamide. The mechanism of action is membrane stabilisation, which can either be achieved by blockade of chloride and bicarbonate membrane transport as it is proposed in epilepsy or by producing kaliuresis and raising the transmembrane potential by decreasing extracellular potassium as it is proposed in acetazolamide responsive myotonia. Whatever the mechanism is, acetazolamide can 
be an alternative drug in continuous muscle fibre activity.

1 Isaacs $\mathrm{H}$. A syndrome of continuous muscle fibre activity. $\mathcal{F}$ Neurol Neurosurg Psychiatry 1961;24:319-25.

2 Isaacs $\mathrm{H}$. Continuous muscle fibre activity in an Indian male with additional evidence of terminal motor fiber abnormality. $\mathcal{F}$ Neurol Neurosurg Psychiatry 1967;30:126-33.

3 Isaacs $\mathrm{H}$, Hefron JJA. The syndrome of continuous muscle fibre activity cured: further studies. $\mathcal{F}$ Neurol Neurosurg Psychiatry 1974;37:1231-5

4 Catteral WA. Common modes of drug action on sodium channels: local anesthetics, antiarhytmics and anticonvulsants. Trends Pharmacol Sci 1987;8:57-65.

5 Shorvon S. The drug treatment of epilepsy. In: Hopkins A Shorvon S, Casino G, eds. Epilepsy. London: Chapman and Hall Medical, 1995:171-214.

6 Griggs RC, Engel WK, Resnick JJ. Acetozolamide treatment of hyperkalaemik periodic paralysis. Ann Intern Med 1970; 73:39-48.

7 Vroom FQ, Jorrell MA, Maren TH. Acetozolamide treatment of hypokalaemic periodic paralysis: Probable treatment of hypokalaemic periodic paralysis: Prob

8 Jarrell MA, Greer M, Maren TH. The effect of acidosis in hypokalaemic periodic paralysis. Arch Neurol 1976;33:791-

9 Riggs JE, Griggs RC, Moxley RT. Dissociation of glucose and potassium arterial-venous differences across the forearm by acetozolamide. Arch Neurol 1984;41:35-8.
10 Trudell RG, Kaiser KK, Griggs RC. Acetozolamide responsive myotonia congenita. Neurology 1987;37:488-91.

11 Ptacek LJ, Tawil R, Griggs RC, et al. Sodium channel mutations in acetozolamide-responsive myotonia congenita, paramyotonia congenita and hyperkalaemic periodic paralysis. Neurology 1994;44:1500-3.

12 Brunt ERP, Van Weerden TW. Familial paroxsymal kinesigenic ataxia and continuous myokymia. Brain 1990; 113:1361-82.

13 Vaamonde J, Artieda J, Obeso JA. Hereditary paroxysmal ataxia with neuromyotonia. Mov Disord 1991;6:180-2.

14 Browne DL, Gancher ST,Nutt JG, et al. Episodic ataxia/ myokymia syndrome is associated with point mutations in the human potassium channel gene, KCNA 1. Nat Genet 1994;8:136-40.

15 Sinha S, Newsom-Davis J, Mills K, Byrne N, Lang B, Vincent A. Autoimmune aetiology for aquired neuromyotonia (Isaacs' syndrome). Lancet 1991;338:75-7.

16 Newsom-Davis J, Mills KR. Immunological associations of aquired neuromyotonia (Isaacs' syndrome): report of 5 cases and literature review. Brain 1993;116:453-69.

17 Shillito P, Molenaar PC, Vincent A, et al. Aquired neuromyotonia: evidence for antibodies directed against potassium channels of peripheral nerves. Ann Neurol 1995; 38:714-22.

18 Wasserstein PH, Shadlen MN, Dolfman LJ Succesful treatment of neuromyotonia with mexiletine [abstract]. Neurology 1992;42(suppl 3):270.

19 Mertens HG, Zschoke S. Neuromyotonie. Klin Wochenschr $1965 ; 47: 280-7$. 